NRCPS-HE-10-17

\title{
Extension of the Poincaré Group \\ and \\ Non-Abelian Tensor Gauge Fields
}

\author{
George Savvidy \\ Demokritos National Research Center \\ Institute of Nuclear Physics \\ Ag. Paraskevi, GR-15310 Athens, Greece
}

\begin{abstract}
In the recently proposed generalization of the Yang-Mills theory the group of gauge transformation gets essentially enlarged. This enlargement involves an elegant mixture of the internal and space-time symmetries. The resulting group is an extension of the Poincaré group with infinitely many generators which carry internal and space-time indices. This is similar to the super-symmetric extension of the Poincaré group, where instead of an anti-commuting spinor variable one should introduce a new vector variable.

The construction of irreducible representations of the extended Poincaré algebra identifies a vector variable with the derivative of the Pauli-Lubanski vector over its length. As a result of this identification the generators of the gauge group have nonzero components only in the plane transversal to the momentum and are projecting out non-Abelian tensor gauge fields into the transversal plane, keeping only their positively definite space-like components.
\end{abstract}




\section{Introduction}

In the recently proposed generalization of the Yang-Mills theory the group of gauge transformation gets essentially enlarged $[4,5,6]$. This enlargement involves an elegant mixture of the internal and space-time symmetries. The resulting group is an extension of the Poincaré group with infinitely many generators which carry internal and space-time indices. $[7,8]$. This is similar to the super-symmetric extension of the Poincaré group $[9,10,11,12,13]$, where instead of the anti-commuting spinor variable $\theta_{\alpha}$ one should introduce a new commuting vector variable $e^{\mu}[20,21,22]$. Our main concern in this article is to study the irreducible representation of this group and its relation with the non-Abelian tensor gauge fields.

Let us first overview the gauge transformations which are defined for the non-Abelian tensor gauge fields and are the source of the proposed extension of the Poincaré group. The non-Abelian gauge fields are defined as rank- $(s+1)$ tensors $[4,5,6]$

$$
A_{\mu \lambda_{1} \ldots \lambda_{s}}^{a}(x), \quad s=0,1,2, \ldots
$$

and are totally symmetric with respect to the indices $\lambda_{1} \ldots \lambda_{s}$. The index $a$ numerates the matrix generators $L_{a}$ of the Lie algebra $L(G)$. The extended non-Abelian gauge transformations $\delta_{\xi} A_{\mu \lambda_{1} \ldots \lambda_{s}}^{a}(x)$ of the tensor gauge fields form a closed group in which gauge parameters are totally symmetric tensors $\xi_{\lambda_{1} \ldots \lambda_{s}}^{a}(x)[4,5,6]$. The extended gauge transformations form a group $\mathcal{P}$ which is in fact a mixture of the space-time and internal symmetries $[7,8]$. Indeed, the commutator of two such gauge transformations can be expressed in the form $[4,5,6]$

$$
\left[\delta_{\eta}, \delta_{\xi}\right] A_{\mu \lambda_{1} \lambda_{2} \ldots \lambda_{s}}=g \delta_{\zeta} A_{\mu \lambda_{1} \lambda_{2} \ldots \lambda_{s}},
$$

where the gauge parameters $\zeta_{\lambda_{1} \ldots \lambda_{s}}^{a}(x)$ are defined by the following equations:

$$
\begin{aligned}
\zeta^{a} & =f^{a b c} \eta^{b} \xi^{c} \\
\zeta_{\lambda_{1}}^{a} & =f^{a b c}\left(\eta^{b} \xi_{\lambda_{1}}^{c}+\eta_{\lambda_{1}}^{b} \xi^{c}\right) \\
\zeta_{\lambda_{1} \lambda_{2}}^{a} & =f^{a b c}\left(\eta^{b} \xi_{\lambda_{1} \lambda_{2}}^{c}+\eta_{\lambda_{1}}^{b} \xi_{\lambda_{2}}^{c}+\eta_{\lambda_{2}}^{b} \xi_{\lambda_{1}}^{c}+\eta_{\lambda_{1} \lambda_{2}}^{b} \xi^{c}\right), \\
\ldots \ldots & . \cdots \ldots \ldots \ldots \ldots \ldots \ldots \ldots \ldots \ldots \ldots \ldots \ldots \ldots \ldots \ldots
\end{aligned}
$$

This is the algebra of our main concern. Because the gauge parameters $\xi_{\lambda_{1} \ldots \lambda_{s}}^{a}(x)$ have internal and space-time indices they transform nontrivially under both groups. To grasp the structure of this algebra let us consider the first line of the above equation. It encodes the structure of the internal Lie algebra $\left[L_{a}, L_{b}\right]=i f_{a b c} L_{c}$ and we have to ask: what is the structure of the algebra which is behind the rest of the equations? Let us introduce for that a translationally invariant commuting vector variable $e_{\lambda}$ and define an infinite set of new generators as follows [6]:

$$
L_{a}^{\lambda_{1} \ldots \lambda_{s}}=e^{\lambda_{1}} \ldots e^{\lambda_{s}} \otimes L_{a} \quad s=0,1,2 \ldots \ldots
$$

These generators carry space-time and internal indices and transform under the operations of both groups. The algebra of these generators [6]

$$
\left[L_{a}^{\lambda_{1} \ldots \lambda_{i}}, L_{b}^{\lambda_{i+1} \ldots \lambda_{s}}\right]=i f_{a b c} L_{c}^{\lambda_{1} \ldots \lambda_{s}}, \quad s=0,1,2 \ldots
$$


encodes all equations (1) into a universal one $\zeta(L)=-i[\eta(L), \xi(L)]$, where the gauge parameters are unified into one function $\zeta(x, L)=\sum_{s} \frac{1}{s !} \zeta_{\lambda_{1} \ldots \lambda_{s}}^{a}(x) L_{a}^{\lambda_{1} \ldots \lambda_{s}}$.

The "current" algebra (1) - (3) is not yet completely defined because it does not specify how new generators $L_{a}^{\lambda_{1} \ldots \lambda_{s}}$ transform under the space-time transformations. Taking into account that the generators $L_{a}^{\lambda_{1} \ldots \lambda_{s}}$ are translationally invariant tensors ${ }^{1}$ of the rank s one can suggest the following extension of the Poincaré algebra $[7,8]$ :

$$
\begin{aligned}
& {\left[P^{\mu}, P^{\nu}\right]=0} \\
& {\left[M^{\mu \nu}, P^{\lambda}\right]=i\left(\eta^{\lambda \nu} P^{\mu}-\eta^{\lambda \mu} P^{\nu}\right)} \\
& {\left[M^{\mu \nu}, M^{\lambda \rho}\right]=i\left(\eta^{\mu \rho} M^{\nu \lambda}-\eta^{\mu \lambda} M^{\nu \rho}+\eta^{\nu \lambda} M^{\mu \rho}-\eta^{\nu \rho} M^{\mu \lambda}\right)} \\
& {\left[P^{\mu}, L_{a}^{\lambda_{1} \ldots \lambda_{s}}\right]=0} \\
& {\left[M^{\mu \nu}, L_{a}^{\lambda_{1} \ldots \lambda_{s}}\right]=i\left(\eta^{\lambda_{1} \nu} L_{a}^{\mu \lambda_{2} \ldots \lambda_{s}}-\ldots-\eta^{\lambda_{s} \mu} L_{a}^{\lambda_{1} \ldots \lambda_{s}-1 \nu}\right),} \\
& {\left[L_{a}^{\lambda_{1} \ldots \lambda_{i}}, L_{b}^{\lambda_{i+1} \ldots \lambda_{s}}\right]=i f_{a b c} L_{c}^{\lambda_{1} \ldots \lambda_{s}} \quad(\mu, \nu, \rho, \lambda=0,1,2,3 ; \quad s=0,1,2, \ldots)}
\end{aligned}
$$

The first three commutators define the Poincaré algebra as its subalgebra. The next two commutators tell that the generators $L_{a}^{\lambda_{1} \ldots \lambda_{s}}$ are translationally invariant tensors of rank s and the last commutator defines the "current" subalgebra (3). One can check that all Jacoby identities are satisfied and we have an example of fully consistent algebra, which we shall call an extended Poincaré algebra $L(\mathcal{P})$ associated with a compact Lie group $G$.

As far as the algebra is formulated one can now abstract oneself from the path which led to it and begin studing its properties and representations. First of all it is a "gauge invariant" extension of the Poincaré algebra in a sense that if one defines a "gauge" transformation of its generators as

$$
L_{a}^{\lambda_{1} \ldots \lambda_{s}} \rightarrow L_{a}^{\lambda_{1} \ldots \lambda_{s}}+\sum_{1} P^{\lambda_{1}} L_{a}^{\lambda_{2} \ldots \lambda_{s}}
$$

then one can check that the algebra $L(\mathcal{P})$ remains intact. The second important property of the algebra is that it is consistent with the "divergentless" condition imposed on the generators:

$$
P_{\lambda_{1}} L_{a}^{\lambda_{1} \ldots \lambda_{s}}=0
$$

As we shall see, these equations are automatically fulfilled for the matrix representations which we shall construct in the next sections. This extension of the Poincaré algebra is also consistent with the Coleman-Mandula theorem $[2,3]$ because it has infinitely many generators and states, which are all massless.

The Casimir operators of the algebra include $P^{2}$ and the square of the Pauli-Lubanski vector $w^{\mu}=\frac{1}{2} \epsilon^{\mu \nu \lambda \rho} P_{\nu} M_{\lambda \rho}$. As we shall see, the construction of irreducible representation of the algebra $L(\mathcal{P})$ identifies the translationally invariant vector $e^{\mu}$ with the derivative of the Pauli-Lubanski vector over its length, so that $e^{\mu}=\hat{w}^{\mu}$ (see Appendix A for details) and the representation takes the form

$$
L_{a}^{\perp \lambda_{1} \ldots \lambda_{s}}=\hat{w}^{\lambda_{1}} \ldots \hat{w}^{\lambda_{s}} \otimes L_{a} .
$$

These symmetric generators are transversal $P_{\lambda_{1}} L_{a}^{\perp \lambda_{1} \ldots \lambda_{s}}=0$, space-like tensors carrying the helicities:

$$
\pm s, \quad \pm(s-2), \quad \pm(s-4), \ldots
$$

\footnotetext{
${ }^{1}$ They inherited this property from the vector variable $e^{\mu}$ in (2).
} 
because each vector $\hat{w}^{\lambda_{i}}$ is a transversal $P_{\mu} \hat{w}^{\mu}=0$ and purely spatial unit vector $\hat{w}^{2}=-1$, which carries a non-zero helicities $h= \pm 1^{2}$.

Therefore the generators $L_{a}^{\perp \lambda_{1} \ldots \lambda_{s}}$ are projecting out the components of the non-Abelian tensor gauge field $A_{\mu \lambda_{1} \ldots \lambda_{s}}^{a}$ into the plane transversal to the momentum:

$$
A_{\mu \lambda_{1} \ldots \lambda_{s}}^{a} L_{a}^{\perp \lambda_{1} \ldots \lambda_{s}}
$$

keeping only its positively definite space-like components of helicities:

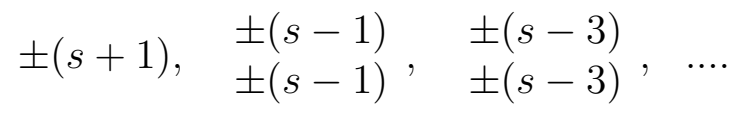

where the lower helicity states have double degeneracy. These are the main results.

We have also found that the current subalgebra (3) allows "central-like" extension of the form

$$
\begin{aligned}
& {\left[L_{a}^{\lambda_{1}}, L_{b}^{\lambda_{2}}\right]=i f_{a b c} L_{c}^{\lambda_{1} \lambda_{2}}-\frac{i}{4} \delta_{a b} \epsilon^{\lambda_{1} \lambda_{2} \rho \sigma} w_{\rho} P_{\sigma}} \\
& \begin{aligned}
{\left[L_{a}^{\lambda_{1} \lambda_{2}}, L_{b}^{\lambda_{3}}\right]=i f_{a b c} L_{c}^{\left(\lambda_{1} \lambda_{2}\right) \lambda_{3}}+\frac{i}{4} \delta_{a b} } & \left(\epsilon^{\lambda_{1} \lambda_{3} \rho \sigma}\left\{w^{\lambda_{2}} w_{\rho}\right\}+\epsilon^{\lambda_{2} \lambda_{3} \rho \sigma}\left\{w^{\lambda_{1}} w_{\rho}\right\}\right) P_{\sigma} \\
{\left[L_{a}^{\lambda_{1} \lambda_{2}}, L_{b}^{\lambda_{3} \lambda_{4}}\right]=i f_{a b c} L_{c}^{\left(\lambda_{1} \lambda_{2}\right)\left(\lambda_{3} \lambda_{4}\right)}-} & \frac{i}{4} \delta_{a b}\left(\epsilon^{\lambda_{1} \lambda_{3} \rho \sigma}\left\{w^{\lambda_{2}}\left\{w_{\rho} w^{\lambda_{4}}\right\}\right\}+\epsilon^{\lambda_{2} \lambda_{3} \rho \sigma}\left\{w^{\lambda_{1}}\left\{w_{\rho} w^{\lambda_{4}}\right\}\right\}\right. \\
& \left.+\epsilon^{\lambda_{1} \lambda_{4} \rho \sigma}\left\{w^{\lambda_{2}}\left\{w_{\rho} w^{\lambda_{3}}\right\}\right\}+\epsilon^{\lambda_{2} \lambda_{4} \rho \sigma}\left\{w^{\lambda_{1}}\left\{w_{\rho} w^{\lambda_{3}}\right\}\right\}\right) P_{\sigma}
\end{aligned}
\end{aligned}
$$

The deformation (9) of the current subalgebra (3) appears when the vector $e^{\mu}$ is associated with the Pauli-Lubanski vector $e^{\mu}=w^{\mu}$, which is a noncommutative vector operator (42). The derivative of the Pauli-Lubanski vector over its length $\hat{w}^{\nu}$ - is a commutative vector operator (49) and this is the reason why the corresponding current subalgebra for the transversal generators (6) is without anomalous terms. The appearance of the momentum operator in the commutators between tensor generators $L_{a}^{\lambda_{1} \ldots \lambda_{s}}$ in this deformed subalgebra is reminiscent of the super-Poincaré algebra (18).

In conclusion we shall discuss a possible geometrical interpretation of the group $\mathcal{P}$ and of the corresponding gauge field theory $[4,5,6]$. In the extended Yang-Mills theory, with each space-time point $\mathrm{x}$ one should associate a vector space $\mathcal{V}_{x}$ of charged RaritaSchwinger tensor-spinors fields $\psi_{\lambda_{1} \ldots \lambda_{s}} \hat{w}^{\lambda_{1}} \ldots \hat{w}^{\lambda_{s}}$, on which the action of the group $\mathcal{P}$ is defined by the group element

$$
U_{\xi}=\exp \left(\sum \frac{1}{s !} \xi_{\lambda_{1} \ldots \lambda_{s}}^{a} L_{a}^{\perp \lambda_{1} \ldots \lambda_{s}}\right)
$$

The extended gauge field is a connection which defines a parallel transport of spinortensors and is a $L(\mathcal{P})$ algebra valued 1-form. The algebra $L(\mathcal{P})$ is not a purely internal algebra - it is a mixture of internal and space-time algebras, which carries not only internal charges, but also a nonzero helicities. The group $\mathcal{P}$ acts simultaneously as a structure group on the fibers and as an isometry group of the base manifold.

\footnotetext{
${ }^{2}$ It is also translationally invariant vector, because $\left[P^{\mu}, \hat{w}^{\nu}\right]=0$.
} 


\section{Extension of the Poincaré Algebra}

As we already explained in the introduction, the gauge fields are defined as rank- $(s+1)$ tensors $[4,5,6]$ (the Abelian fields are considered in $[1,18,19,20,21,22,24,25,26]$ )

$$
A_{\mu \lambda_{1} \ldots \lambda_{s}}^{a}(x), \quad s=0,1,2, \ldots
$$

and are totally symmetric with respect to the indices $\lambda_{1} \ldots \lambda_{s}$. A priory the tensor fields have no symmetries with respect to the first index $\mu$. The index $a$ numerates the generators $L^{a}$ of the Lie algebra $L(G)$.

One can think of these tensor fields as appearing in the expansion of the extended gauge field $\mathcal{A}_{\mu}(x, L)$ over the generators $L_{a}^{\lambda_{1} \ldots \lambda_{s}}[6]$ :

$$
\mathcal{A}_{\mu}(x, L)=\sum_{s=0}^{\infty} \frac{1}{s !} A_{\mu \lambda_{1} \ldots \lambda_{s}}^{a}(x) L_{a}^{\lambda_{1} \ldots \lambda_{s}} .
$$

The gauge field $A_{\mu \lambda_{1} \ldots \lambda_{s}}^{a}$ carries indices $a, \lambda_{1}, \ldots, \lambda_{s}$ labeling the generators of the extended Poincaré algebra $L(\mathcal{P})$ associated with a compact Lie group $G$. It has infinitely many generators $L_{a}^{\lambda_{1} \ldots \lambda_{s}}$ and the corresponding "current" algebra is given by the commutator [6]:

$$
\left[L_{a}^{\lambda_{1} \ldots \lambda_{i}}, L_{b}^{\lambda_{i+1} \ldots \lambda_{s}}\right]=i f_{a b c} L_{c}^{\lambda_{1} \ldots \lambda_{s}} .
$$

Because the generators $L_{a}^{\lambda_{1} \ldots \lambda_{s}}$ are space-time tensors, they do not commute with the generators of the Poincaré algebra $P^{\mu}, M^{\mu \nu}$. They act on the space-time components of the above generators as follows $[7,8]$ :

$$
\begin{aligned}
& {\left[P^{\mu}, P^{\nu}\right]=0,} \\
& {\left[M^{\mu \nu}, P^{\lambda}\right]=i\left(\eta^{\lambda \nu} P^{\mu}-\eta^{\lambda \mu} P^{\nu}\right),} \\
& {\left[M^{\mu \nu}, M^{\lambda \rho}\right]=i\left(\eta^{\mu \rho} M^{\nu \lambda}-\eta^{\mu \lambda} M^{\nu \rho}+\eta^{\nu \lambda} M^{\mu \rho}-\eta^{\nu \rho} M^{\mu \lambda}\right),} \\
& {\left[P^{\mu}, L_{a}^{\lambda_{1} \ldots \lambda_{s}}\right]=0,} \\
& {\left[M^{\mu \nu}, L_{a}^{\lambda_{1} \ldots \lambda_{s}}\right]=i\left(\eta^{\lambda_{1} \nu} L_{a}^{\mu \lambda_{2} \ldots \lambda_{s}}-\eta^{\lambda_{1} \mu} L_{a}^{\nu \lambda_{2} \ldots \lambda_{s}}+\ldots+\eta^{\lambda_{s} \nu} L_{a}^{\lambda_{1} \ldots \lambda_{s-1} \mu}-\eta^{\lambda_{s} \mu} L_{a}^{\lambda_{1} \ldots \lambda_{s-1} \nu}\right),} \\
& {\left[L_{a}^{\lambda_{1} \ldots \lambda_{i}}, L_{b}^{\lambda_{i+1} \ldots \lambda_{s}}\right]=i f_{a b c} L_{c}^{\lambda_{1} \ldots \lambda_{s}} \quad(\mu, \nu, \rho, \lambda=0,1,2,3 ; \quad s=0,1,2, \ldots),}
\end{aligned}
$$

where the flat space-time metric is $\eta^{\mu \nu}=\operatorname{diag}(+1,-1,-1,-1)$. One can check that all Jacoby identities are satisfied and we have an example of a fully consistent algebra. The commutation relations of the Lorentz generators $M^{\mu \nu}$ correspond to the pseoudoorthogonal algebra $S O(1,3)$. The algebra $L(\mathcal{P})$ incorporates the Poincaré algebra and an internal algebra $L(G)$ in a nontrivial way, which is different from the direct product. Specifically the generators $L_{a}^{\lambda_{1} \ldots \lambda_{s}}$ have a nonzero commutation relation with $M^{\mu \nu}$, which means that the generators of this new symmetry have nontrivial Lorentz transformation and that

they have a spin different from zero. They will then relate states of different spins. The algebra is invariant with respect to the following gauge transformations:

$$
\begin{aligned}
& L_{a}^{\lambda_{1} \ldots \lambda_{s}} \rightarrow L_{a}^{\lambda_{1} \ldots \lambda_{s}}+\sum_{1} P^{\lambda_{1}} L_{a}^{\lambda_{2} \ldots \lambda_{s}} \\
& M^{\mu \nu} \rightarrow M^{\mu \nu}, \quad P^{\lambda} \rightarrow P^{\lambda},
\end{aligned}
$$


where the sum $\sum_{1}$ is over all inequivalent index permutations.

It is worthy to compare the above extension of the Poincaré algebra with the superPoincaré algebra which is defined as follows $[9,10,11,12,13]$ :

$$
\begin{aligned}
& {\left[P^{\mu}, P^{\nu}\right]=0} \\
& {\left[M^{\mu \nu}, P^{\lambda}\right]=i\left(\eta^{\lambda \nu} P^{\mu}-\eta^{\lambda \mu} P^{\nu}\right)} \\
& {\left[M^{\mu \nu}, M^{\lambda \rho}\right]=i\left(\eta^{\mu \rho} M^{\nu \lambda}-\eta^{\mu \lambda} M^{\nu \rho}+\eta^{\nu \lambda} M^{\mu \rho}-\eta^{\nu \rho} M^{\mu \lambda}\right),} \\
& {\left[P^{\mu}, \Theta_{\alpha}^{i}\right]=0} \\
& {\left[M^{\mu \nu}, \Theta_{\alpha}^{i}\right]=\frac{i}{2}\left(\gamma^{\mu \nu} \Theta^{i}\right)_{\alpha}, \quad \gamma^{\mu \nu}=\frac{1}{2}\left[\gamma^{\mu}, \gamma^{\nu}\right]} \\
& \left\{\Theta_{\alpha}^{i}, \Theta_{\beta}^{j}\right\}=-2 \delta^{i j}\left(\gamma^{\mu} C\right)_{\alpha \beta} P_{\mu}, \quad i=1, \ldots, N
\end{aligned}
$$

Both algebras have Poincaré algebra (12), (16) as subalgebra. The next two commutators (12) and (17) express the fact that the extended generators $\Theta_{\alpha}^{i}$ and $L_{a}^{\lambda_{1} \ldots \lambda_{s}}$ are translationally invariant operators and carry a nonzero spin. The last commutators (14) and (18) are essentially different in both of the algebras, in super-Poincaré algebra the generators $\Theta_{\alpha}^{i}$ anti-commute to the operator $P^{\mu}$, while in our case $L_{a}^{\lambda_{1} \ldots \lambda_{s}}$ commute to themselves forming an infinite series of commutators of "current" subalgebra (11) which cannot be truncated, so that the index $s$ runs from zero to infinity. We have here an example of an infinitely dimensional current subalgebra [16].

The algebra $L(\mathcal{P})$ has a simple representation of the following form:

$$
\begin{aligned}
& P^{\mu}=k^{\mu} \\
& M^{\mu \nu}=i\left(k^{\mu} \frac{\partial}{\partial k_{\nu}}-k^{\nu} \frac{\partial}{\partial k_{\mu}}\right)+i\left(e^{\mu} \frac{\partial}{\partial e_{\nu}}-e^{\nu} \frac{\partial}{\partial e_{\mu}}\right), \\
& L_{a}^{\lambda_{1} \ldots \lambda_{s}}=e^{\lambda_{1}} \ldots e^{\lambda_{s}} \otimes L_{a},
\end{aligned}
$$

therefore it has at least one nontrivial representation. This representation appeared, when we were considering the transformation properties of the non-Abelian tensor gauge fields $[4,5,6]$. Our aim now is to study the matrix representations of this algebra. This will allow to specify the properties of the vector variable $e^{\mu}$ and to identify it with the derivative of the Pauli-Lubanski vector over its length - $e^{\mu}=\hat{w}^{\mu}$ (see Appendix A for details). This vector has all required properties:

$\alpha)$ it is a commuting vector: $\left[\hat{w}^{\mu}, \hat{w}^{\nu}\right]=0$,

$\beta)$ it is translationally invariant: $\left[P^{\mu}, \hat{w}^{\nu}\right]=0$,

$\gamma)$ it is a transversal vector: $P^{\mu} \hat{w}_{\mu}=0$,

$\delta$ ) it is a unit space-like vector: $\hat{w}^{2}=-1$,

so that the corresponding generators

$$
L_{a}^{\perp \lambda_{1} \ldots \lambda_{s}}=\hat{w}^{\lambda_{1}} \ldots \hat{w}^{\lambda_{s}} \otimes L_{a}
$$

are transversal tensors, because $P^{\mu} \hat{w}_{\mu}=0$ and $\hat{w}^{2}=-1$, projecting out the components of non-Abelian tensor gauge field into the plane transversal to the momentum $A_{\mu \lambda_{1} \ldots \lambda_{s}}^{a} L_{a}^{\perp \lambda_{1} \ldots \lambda_{s}}$, keeping only their positively definite space-like components. 


\section{Matrix Representations of Extended Poincaré Algebra}

The irreducible representation of the Poincaré subalgebra (12), (16) can be found by the method of induced representations [23]. This method consists of finding a representation of the subgroup of the Poincaré group and boosting it up to a representation of the full group. One should choose a given momentum $k^{\mu}=\omega(1,0,0,1)$ which satisfies $k^{2}=0$ and then find the subgroup $\mathrm{H}$ which leaves $k^{\mu}$ intact and find a representation of $\mathrm{H}$ on the $\mid k^{\mu}>$ states. One should then induce this representation of $\mathrm{H}$ to the whole Poincaré group by boosting the frame with momentum $k^{\mu}$ to the one with an arbitrary momentum. One can show that the result is independent of the choice of momentum $k^{\mu}$ [23].

We must find $\mathrm{H}$, the group elements of which leave $k^{\mu}=\omega(1,0,0,1)$ intact. Under the Lorentz group the action of the element

$$
U_{\theta}=\exp \left(\frac{i}{2} \theta_{\mu \nu} M^{\mu \nu}\right)
$$

creates an infinitesimal transformation $k^{\mu} \rightarrow \theta^{\mu}{ }_{\nu} k^{\nu}+k^{\mu}$. Hence $k^{\mu}=\omega(1,0,0,1)$ is left invariant provided the parameters obey the relations

$$
\theta_{30}=0, \quad \theta_{10}+\theta_{13}=0, \quad \theta_{20}+\theta_{23}=0,
$$

hence the Lorentz and $L_{a}^{\lambda_{1} \ldots \lambda_{s}}$ generators in subalgebra $\mathrm{H}$ are

$$
M^{12}, \quad \Pi^{\prime}=-M^{10}+M^{13}, \quad \Pi^{\prime \prime}=-M^{20}+M^{23}, \quad L_{a}^{\lambda_{1} \ldots \lambda_{s}}
$$

with commutators [23]

$$
\left[\Pi^{\prime}, \Pi^{\prime \prime}\right]=0, \quad\left[M^{12}, \Pi^{\prime}\right]=i \Pi^{\prime \prime}, \quad\left[M^{12}, \Pi^{\prime \prime}\right]=-i \Pi^{\prime} .
$$

We have to study the commutation relations between $M_{12}, \Pi^{\prime}, \Pi^{\prime \prime}$ and $L_{a}^{\lambda_{1} \ldots \lambda_{s}}$. First of all, the relations

$$
\left[P^{\mu}, L_{a}\right]=0, \quad\left[M^{\mu \nu}, L_{a}\right]=0
$$

show that the generators of the Poincaré group commute with the generators of the internal algebra $\mathrm{L}(\mathrm{G})$, as they should [2]. The next level commutation relation is

$$
\left[M^{\mu \nu}, L_{a}^{\lambda}\right]=i\left(\eta^{\lambda \nu} L_{a}^{\mu}-\eta^{\lambda \mu} L_{a}^{\nu}\right)
$$

and it provides the structure of the subgroup $\mathrm{H}$ with the rotation $M^{12}$ :

$$
\begin{array}{ll}
{\left[M^{12},\right.} & \left.L_{a}^{0}\right]=\left[M^{12}, L_{a}^{3}\right]=0 \\
{\left[M^{12},\right.} & \left.L_{a}^{1}\right]=+i L_{a}^{2} \\
{\left[M^{12},\right.} & \left.L_{a}^{2}\right]=-i L_{a}^{1},
\end{array}
$$

and with translation operators $\Pi^{\prime}$ and $\Pi^{\prime \prime}$ :

$$
\begin{array}{ll}
{\left[\Pi^{\prime}, L_{a}^{0}\right]=-i L_{a}^{1}} & {\left[\Pi^{\prime \prime}, L_{a}^{0}\right]=-i L_{a}^{2}} \\
{\left[\Pi^{\prime}, L_{a}^{3}\right]=-i L_{a}^{1}} & {\left[\Pi^{\prime \prime}, L_{a}^{3}\right]=-i L_{a}^{2}} \\
{\left[\Pi^{\prime}, L_{a}^{1}\right]=-i\left(L_{a}^{0}-L_{a}^{3}\right)} & {\left[\Pi^{\prime \prime}, L_{a}^{1}\right]=0} \\
{\left[\Pi^{\prime}, L_{a}^{2}\right]=0} & {\left[\Pi^{\prime \prime}, L_{a}^{2}\right]=-i\left(L_{a}^{0}-L_{a}^{3}\right) .}
\end{array}
$$

The equations (23),(25) and (26) define the first level generators $L_{a}^{\lambda}$. Our aim is to find the matrix solution of the equations $(23),(25)$ and (26). 


\subsection{Longitudinal Representation}

If the representation of the algebra (23) has $\varrho=0$ and $\Pi^{\prime}=\Pi^{\prime \prime}=0$, the so called $\mathrm{O}_{\mathrm{s}}$ representations (see Appendix A), then from (26) it follows that $L_{a}^{1}, L_{a}^{2}$ are trivial operators. The only nonzero operators are $M^{12}, L_{a}^{0}, L_{a}^{3}$ :

$$
M^{12}=\sigma \otimes 1, \quad L_{a}^{0}=\sigma \otimes L_{a}, \quad L_{a}^{3}=\sigma \otimes L_{a},
$$

where $\sigma$ is an integer diagonal matrix and we are using the Kronecker product (see Appendix B) and the representation (67). This representation is purely longitudinal because the transversal components $L_{a}^{1}=L_{a}^{2}=0$ of the generator $L_{a}^{\lambda}$ vanish. The next level operators can be easily constructed by computing commutators between low level generators, as in (14), that gives:

$$
L_{a}^{00}=L_{a}^{03}=L_{a}^{33}=\sigma^{2} \otimes \tau_{a}, \quad L_{a}^{01}=L_{a}^{02}=L_{a}^{13}=L_{a}^{11}=L_{a}^{22}=L_{a}^{23}=0
$$

and so on. This representation is isomorphic to the representation considered in the previous section (2) with the vector variable $e^{\lambda}$ having only longitudinal components $e^{\lambda}=k^{\lambda}$, so that the generators take the form

$$
L_{a}^{\| \lambda_{1} \ldots \lambda_{s}}=k^{\lambda_{1}} \ldots k^{\lambda_{s}} \otimes L_{a}
$$

and are obviously divergentless tensors (5). Our aim is to find out representations for the generators $L_{a}^{\lambda_{1} \ldots \lambda_{s}}$ which have only transversal components.

\subsection{Transversal Representation}

In order to get different representations which shall have nonzero transversal components we are going to take the infinitely dimensional representation $\mathbf{O}(\varrho)$ of the algebra (23) which has $\varrho \neq 0$ (see Appendix A). We shall take it in the form of Kronecker product with the identity matrix in the vector space of representations of the internal algebra G:

$$
\begin{aligned}
& M^{12}=+m_{12} \otimes 1 \\
& \Pi^{\prime}=+\pi^{\prime} \otimes 1 \\
& \Pi^{\prime \prime}=+\pi^{\prime \prime} \otimes 1 .
\end{aligned}
$$

Because $L_{a}^{0}$ and $L_{a}^{3}$ commute with $M^{12}$, we shall take them proportional to $m^{12}$. The other commutators in (25) tell us that $L_{a}^{1}$ and $L_{a}^{2}$ are proportional to $\pi^{\prime}$ and $\pi^{\prime \prime}$. We shall take them also in the form of Kronecker product with the same matrix representation $L_{a}$ of the internal algebra $L(G)$ in order to get the following representation of the extended little algebra:

$$
\begin{aligned}
& L_{a}^{1}=+\pi^{\prime \prime} \otimes L_{a} \\
& L_{a}^{2}=-\pi^{\prime} \otimes L_{a} \\
& L_{a}^{0}=+m_{12} \otimes L_{a} \\
& L_{a}^{3}=+m_{12} \otimes L_{a} .
\end{aligned}
$$

It is easy to check that it fulfills all the commutation relations (25) and (26) and has nonzero transversal components $L_{a}^{1}$ and $L_{a}^{2}$. One can see also that divergentless equation (5) is satisfied:

$$
k_{\lambda} L^{\lambda}=L_{a}^{0}-L_{a}^{3}=0,
$$


so that the vector generator $L_{a}^{\lambda}$ is orthogonal to the momentum $k^{\mu}$ and cannot be time-like.

Our next task is to construct generators on the second level. The second level commutation relation is

$$
\left[M^{\mu \nu}, L_{a}^{\lambda_{1} \lambda_{2}}\right]=i\left(\eta^{\lambda_{1} \nu} L_{a}^{\mu \lambda_{2}}-\eta^{\lambda_{1} \mu} L_{a}^{\nu \lambda_{2}}+\eta^{\lambda_{2} \nu} L_{a}^{\mu \lambda_{1}}-\eta^{\lambda_{2} \mu} L_{a}^{\nu \lambda_{1}}\right)
$$

and it provides the structure of the subgroup with rotation $M^{12}$ :

$$
\begin{array}{ll}
{\left[M^{12}, L_{a}^{00}\right]} & =\left[M^{12}, L_{a}^{03}\right]=\left[M^{12}, L_{a}^{33}\right]=0 \\
{\left[M^{12},\right.} & \left.L_{a}^{01}\right]=+i L_{a}^{02} \\
{\left[M^{12},\right.} & \left.L_{a}^{02}\right]=-i L_{a}^{01} \\
{\left[M^{12}, L_{a}^{13}\right]=+i L_{a}^{23}} \\
{\left[M^{12}, L_{a}^{23}\right]=-i L_{a}^{13}} \\
{\left[M^{12}, L_{a}^{11}\right]=+2 i L_{a}^{12}} \\
{\left[M^{12}, L_{a}^{22}\right]=-2 i L_{a}^{12}} \\
{\left[M^{12}, L_{a}^{12}\right]=+i\left(L_{a}^{22}-L_{a}^{11}\right)}
\end{array}
$$

and with translation operators $\Pi^{\prime}$ and $\Pi^{\prime \prime}$ :

$$
\begin{array}{lll}
{\left[\Pi^{\prime}, L_{a}^{00}\right]=-2 i L_{a}^{01},} & {\left[\Pi^{\prime \prime}, L_{a}^{00}\right]=-2 i L_{a}^{02}} \\
{\left[\Pi^{\prime}, L_{a}^{01}\right]=-i L_{a}^{00}+i L_{a}^{03}-i L_{a}^{11},} & {\left[\Pi^{\prime \prime}, L_{a}^{01}\right]=-i L_{a}^{12}} \\
{\left[\Pi^{\prime}, L_{a}^{02}\right]=-i L_{a}^{12},} & {\left[\Pi^{\prime \prime}, L_{a}^{02}\right]=-i L_{a}^{00}+i L_{a}^{03}-i L_{a}^{22}} \\
{\left[\Pi^{\prime}, L_{a}^{03}\right]=-i L_{a}^{01}-i L_{a}^{13},} & {\left[\Pi^{\prime \prime}, L_{a}^{03}\right]=-i L_{a}^{02}-i L_{a}^{23}} \\
{\left[\Pi^{\prime}, L_{a}^{11}\right]=-2 i L_{a}^{01}+2 i L_{a}^{13},} & {\left[\Pi^{\prime \prime}, L_{a}^{11}\right]=2 i L_{a}^{23}-2 i L_{a}^{02}} \\
{\left[\Pi^{\prime}, L_{a}^{12}\right]=i L_{a}^{23}-i L_{a}^{02},} & {\left[\Pi^{\prime \prime}, L_{a}^{12}\right]=i L_{a}^{13}-i L_{a}^{01}} \\
{\left[\Pi^{\prime}, L_{a}^{13}\right]=-i L_{a}^{11}+i L_{a}^{33}-i L_{a}^{03},} & {\left[\Pi^{\prime \prime}, L_{a}^{13}\right]=-i L_{a}^{12}} \\
{\left[\Pi^{\prime}, L_{a}^{22}\right]=0,} & {\left[\Pi^{\prime \prime}, L_{a}^{22}\right]=0} \\
{\left[\Pi^{\prime}, L_{a}^{23}\right]=-i L_{a}^{12},} & {\left[\Pi^{\prime \prime}, L_{a}^{23}\right]=-i L_{a}^{22}+i L_{a}^{33}-i L_{a}^{03}} \\
{\left[\Pi^{\prime}, L_{a}^{33}\right]=-2 i L_{a}^{13},} & {\left[\Pi^{\prime \prime}, L_{a}^{33}\right]=-2 i L_{a}^{23}}
\end{array}
$$

The current subalgebra (11) between generators has the following form:

$$
\begin{aligned}
& {\left[L_{a}^{0}, L_{b}^{0}\right]=i f_{a b c} L_{c}^{00},\left[L_{a}^{0}, L_{b}^{1}\right]=i f_{a b c} L_{c}^{01},\left[L_{a}^{0}, L_{b}^{2}\right]=i f_{a b c} L_{c}^{02},\left[L_{a}^{0}, L_{b}^{3}\right]=i f_{a b c} L_{c}^{03}} \\
& {\left[L_{a}^{1}, L_{b}^{1}\right]=i f_{a b c}, L_{c}^{11},\left[L_{a}^{1}, L_{b}^{2}\right]=i f_{a b c} L_{c}^{12},\left[L_{a}^{1}, L_{b}^{3}\right]=i f_{a b c} L_{c}^{13}} \\
& {\left[L_{a}^{2}, L_{b}^{2}\right]=i f_{a b c} L_{c}^{22},\left[L_{a}^{2}, L_{b}^{3}\right]=i f_{a b c} L_{c}^{23}} \\
& {\left[L_{a}^{3}, L_{b}^{3}\right]=i f_{a b c} L_{c}^{33}}
\end{aligned}
$$

Calculating the commutators (35) we can find the representation for the second level generators:

$$
\begin{aligned}
& L_{a}^{11}=+\pi^{\prime \prime} \pi^{\prime \prime} \otimes L_{a} \\
& L_{a}^{12}=-\pi^{\prime} \pi^{\prime \prime} \otimes L_{a} \\
& L_{a}^{22}=+\pi^{\prime} \pi^{\prime} \otimes L_{a} \\
& L_{a}^{00}=L_{a}^{03}=L_{a}^{33}=m_{12} m_{12} \otimes L_{a} \\
& L_{a}^{01}=+\frac{1}{2}\left(m_{12} \pi^{\prime \prime}+\pi^{\prime \prime} m_{12}\right) \otimes L_{a}
\end{aligned}
$$




$$
\begin{aligned}
L_{a}^{02} & =-\frac{1}{2}\left(m_{12} \pi^{\prime}+\pi^{\prime} m_{12}\right) \otimes L_{a} \\
L_{a}^{13} & =+\frac{1}{2}\left(m_{12} \pi^{\prime \prime}+\pi^{\prime \prime} m_{12}\right) \otimes L_{a} \\
L_{a}^{23} & =-\frac{1}{2}\left(m_{12} \pi^{\prime}+\pi^{\prime} m_{12}\right) \otimes L_{a}
\end{aligned}
$$

One can check that all commutators (33) and (34) are satisfied, but what is surprising is that some of the commutators in the current subalgebra (35) are getting additional terms:

$$
\begin{aligned}
& {\left[L_{a}^{0}, L_{b}^{1}\right]=i f_{a b c} L_{c}^{01}-\frac{i}{4} \delta_{a b} \Pi^{\prime},\left[L_{a}^{0}, L_{b}^{2}\right]=i f_{a b c} L_{c}^{02}-\frac{i}{4} \delta_{a b} \Pi^{\prime \prime}} \\
& {\left[L_{a}^{1}, L_{b}^{3}\right]=i f_{a b c} L_{c}^{13}-\frac{i}{4} \delta_{a b} \Pi^{\prime},\left[L_{a}^{2}, L_{b}^{3}\right]=i f_{a b c} L_{c}^{23}-\frac{i}{4} \delta_{a b} \Pi^{\prime \prime}}
\end{aligned}
$$

The appearance of these "central"-like terms 3 is connected with the fact that the matrices $m_{12}$ and $\pi$ in the matrix representation (31) of the vector generator $L_{a}^{\lambda}$ are not commuting. This "central"-type extension of the original algebra $L(\mathcal{P})$ is of the main interest for us, because in this representation of tensor generators $L_{a}^{\lambda_{1} \ldots \lambda_{s}}$ have nontrivial transversal components. As one can check, the above matrix representation also satisfies the divergentless equation

$$
k_{\lambda} L_{a}^{\lambda \mu}=k_{\mu} L_{a}^{\lambda \mu}=0
$$

or, in components,

$$
L_{a}^{00}=L_{a}^{03}=L_{a}^{33}, \quad L_{a}^{01}=L_{a}^{31}, \quad L_{a}^{02}=L_{a}^{32} .
$$

We shall see that also in general

$$
k_{\lambda_{1}} L_{a}^{\lambda_{1} \ldots \lambda_{s}}=0
$$

and therefore tensor generators $L_{a}^{\lambda_{1} \ldots \lambda_{s}}$ are orthogonal to the momentum $k^{\mu}$ and cannot be time-like.

Deformation of the current subalgebra (11) appears also at the third level. Calculating commutators (11) we can find the following sequence of generators:

$$
\begin{aligned}
& L_{a}^{(11) 1}=+\pi^{\prime \prime} \pi^{\prime \prime} \pi^{\prime \prime} \otimes L_{a} \\
& L_{a}^{(11) 2}=L_{a}^{(12) 1}=-\pi^{\prime} \pi^{\prime \prime} \pi^{\prime \prime} \otimes L_{a} \\
& L_{a}^{(12) 2}=L_{a}^{(22) 1}=+\pi^{\prime} \pi^{\prime} \pi^{\prime \prime} \otimes L_{a} \\
& L_{a}^{(22) 2}=-\pi^{\prime} \pi^{\prime} \pi^{\prime} \otimes L_{a} \\
& L_{a}^{(00) 0}=L_{a}^{(00) 3}=L_{a}^{(03) 3}=L_{a}^{(33) 3}=m_{12}^{3} \otimes L_{a} \\
& L_{a}^{(00) 1}=L_{a}^{(03) 1}=L_{a}^{(33) 1}=+\frac{1}{2}\left(m_{12}^{2} \pi^{\prime \prime}+\pi^{\prime \prime} m_{12}^{2}\right) \otimes L_{a} \\
& L_{a}^{(00) 2}=L_{a}^{(03) 2}=L_{a}^{(33) 2}=-\frac{1}{2}\left(m_{12}^{2} \pi^{\prime}+\pi^{\prime} m_{12}^{2}\right) \otimes L_{a} \\
& L_{a}^{(11) 0}=L_{a}^{(11) 3}=+\frac{1}{2}\left(m_{12} \pi^{\prime \prime 2}+\pi^{\prime \prime 2} m_{12}\right) \otimes l_{a} \\
& L_{a}^{(22) 0}=L_{a}^{(22) 3}=+\frac{1}{2}\left(m_{12} \pi^{\prime 2}+\pi^{\prime 2} m_{12}\right) \otimes L_{a}
\end{aligned}
$$

\footnotetext{
${ }^{3}$ In reality it is an operator valued anomaly of the current subalgebra (11), but just for abbreviation we shall call it "central"-like extension or simply deformation of algebra $L(\mathcal{P})$, see also [16].
} 


$$
\begin{aligned}
& L_{a}^{(01) 0}=L_{a}^{(01) 3}=L_{a}^{(31) 0}=L_{a}^{(31) 3}=+\frac{1}{4}\left(m_{12}^{2} \pi^{\prime \prime}+2 m_{12} \pi^{\prime \prime} m_{12}+\pi^{\prime \prime} m_{12}^{2}\right) \otimes L_{a} \\
& L_{a}^{(02) 0}=L_{a}^{(02) 3}=L_{a}^{(32) 0}=L_{a}^{(32) 3}=-\frac{1}{4}\left(m_{12}^{2} \pi^{\prime}+2 m_{12} \pi^{\prime} m_{12}+\pi^{\prime} m_{12}^{2}\right) \otimes L_{a} \\
& L_{a}^{(01) 2}=L_{a}^{(31) 2}=-\frac{1}{4}\left(m_{12} \pi^{\prime} \pi^{\prime \prime}+\pi^{\prime} m_{12} \pi^{\prime \prime}+\pi^{\prime \prime} m_{12} \pi^{\prime}+\pi^{\prime} \pi^{\prime \prime} m_{12}\right) \otimes L_{a} \\
& L_{a}^{(02) 1}=L_{a}^{(32) 1}=-\frac{1}{4}\left(m_{12} \pi^{\prime} \pi^{\prime \prime}+\pi^{\prime} m_{12} \pi^{\prime \prime}+\pi^{\prime \prime} m_{12} \pi^{\prime}+\pi^{\prime} \pi^{\prime \prime} m_{12}\right) \otimes L_{a} \\
& L_{a}^{(01) 1}=L_{a}^{(31) 1}=+\frac{1}{4}\left(m_{12} \pi^{\prime \prime 2}+2 \pi^{\prime \prime} m_{12} \pi^{\prime \prime}+\pi^{\prime \prime} 2 m_{12}\right) \otimes L_{a} \\
& L_{a}^{(02) 2}=L_{a}^{(32) 2}=+\frac{1}{4}\left(m_{12} \pi^{\prime 2}+2 \pi^{\prime} m_{12} \pi^{\prime}+\pi^{\prime 2} m_{12}\right) \otimes L_{a} .
\end{aligned}
$$

What happens is that the generators $L_{a}^{\lambda_{1} \lambda_{2} \lambda_{3}}$ are not any more symmetric over all indices $\lambda_{1} \lambda_{2} \lambda_{3}$, but are symmetric with respect to the following interchanges of indices: $L_{a}^{\left(\lambda_{1} \lambda_{2}\right) \lambda_{3}}=L_{a}^{\left(\lambda_{2} \lambda_{1}\right) \lambda_{3}}=L_{a}^{\lambda_{3}\left(\lambda_{1} \lambda_{2}\right)}$. The "central" terms in the current subalgebra (11) have the following form:

$$
\begin{aligned}
& {\left[L_{a}^{00}, L_{b}^{1}\right]=i f_{a b c} L_{c}^{(00) 1}-\frac{i}{4} \delta_{a b}\left(M^{12} \Pi^{\prime}+\Pi^{\prime} M^{12}\right)} \\
& {\left[L_{a}^{00}, L_{b}^{2}\right]=i f_{a b c} L_{c}^{(00) 2}-\frac{i}{4} \delta_{a b}\left(M^{12} \Pi^{\prime \prime}+\Pi^{\prime \prime} M^{12}\right),} \\
& {\left[L_{a}^{01}, L_{b}^{0}\right]=i f_{a b c} L_{c}^{(01) 0}+\frac{i}{8} \delta_{a b}\left(M^{12} \Pi^{\prime}+\Pi^{\prime} M^{12}\right)} \\
& {\left[L_{a}^{02}, L_{b}^{0}\right]=i f_{a b c} L_{c}^{(02) 0}+\frac{i}{8} \delta_{a b}\left(M^{12} \Pi^{\prime \prime}+\Pi^{\prime \prime} M^{12}\right),} \\
& {\left[L_{a}^{01}, L_{b}^{1}\right]=i f_{a b c} L_{c}^{(01) 1}-\frac{i}{4} \delta_{a b} \Pi^{\prime} \Pi^{\prime \prime}} \\
& {\left[L_{a}^{02}, L_{b}^{2}\right]=i f_{a b c} L_{c}^{(02) 2}+\frac{i}{4} \delta_{a b} \Pi^{\prime} \Pi^{\prime \prime}} \\
& {\left[L_{a}^{01}, L_{b}^{2}\right]=i f_{a b c} L_{c}^{(01) 2}-\frac{i}{4} \delta_{a b} \Pi^{\prime \prime 2}} \\
& {\left[L_{a}^{02}, L_{b}^{1}\right]=i f_{a b c} L_{c}^{(02) 1}+\frac{i}{4} \delta_{a b} \Pi^{\prime 2},} \\
& {\left[L_{a}^{11}, L_{b}^{0}\right]=i f_{a b c} L_{c}^{(11) 0}+\frac{i}{2} \delta_{a b} \Pi^{\prime} \Pi^{\prime \prime}} \\
& {\left[L_{a}^{22}, L_{b}^{0}\right]=i f_{a b c} L_{c}^{(22) 0}-\frac{i}{2} \delta_{a b} \Pi^{\prime} \Pi^{\prime \prime}}
\end{aligned}
$$

In order to construct high rank generators one can proceed in the same way by computing the commutators between the lower rank generators. But already at this stage the general structure of the representation under consideration becomes apparent and one can formulate it in a covariant form using the Pauli-Lubanski vector $w^{\mu}$. This vector is a non-commutative vector and, as we shall see, the above deformation (37), (41) of the current subalgebra (11) appears because of the non-commutativity of this vector. As a next step we shall introduce the vector $\hat{w}^{\mu}$ which is a derivative of the $w^{\mu}$ over its length and is a commutative vector. The corresponding representation of the current subalgebra will be symmetric, transversal and without central-like terms ${ }^{4}$ - the properties of main importance in our formulation of generalization of the Yang-Mills theory.

\footnotetext{
${ }^{4}$ Otherwise the term $\left[\mathcal{A}_{\mu}, \mathcal{A}_{\nu}\right]$ in the field strength tensor will have anomalous terms.
} 


\section{Covariant Form of Transversal Representation}

Our aim is therefore to find the covariant formulas for the above representation. The crucial observation is that the Pauli-Lubanski vector $w^{\mu}=\frac{1}{2} \epsilon^{\mu \nu \lambda \rho} P_{\nu} M_{\lambda \rho}=\frac{1}{2} \epsilon^{\mu \nu \lambda \rho} M_{\nu \lambda} P_{\rho}$ has the following properties:

$$
\left[P^{\mu}, w^{\nu}\right]=0, \quad P^{\mu} w_{\mu}=0, \quad\left[w^{\mu}, w^{\nu}\right]=-i \epsilon^{\mu \nu \lambda \rho} w_{\lambda} P_{\rho}
$$

that is, it is translationally invariant, transversal and non-commutative vector. In components it has the following form (see Appendix A):

$$
w^{\mu}=\left(-m_{12},-\pi^{\prime \prime}, \pi^{\prime},-m_{12}\right) .
$$

This allows to express in a covariant form the first, the second and the third level generators (31), (36) and (40), which we found in the previous section, as follows:

$$
\begin{aligned}
& L_{a}^{\lambda_{1}}=-w^{\lambda_{1}} \otimes L_{a}, \\
& L_{a}^{\lambda_{1} \lambda_{2}}=\frac{1}{2}\left(w^{\lambda_{1}} w^{\lambda_{2}}+w^{\lambda_{2}} w^{\lambda_{1}}\right) \otimes L_{a}, \\
& L_{a}^{\left(\lambda_{1} \lambda_{2}\right) \lambda_{3}}=\frac{1}{4}\left(w^{\lambda_{1}} w^{\lambda_{2}} w^{\lambda_{3}}+w^{\lambda_{2}} w^{\lambda_{1}} w^{\lambda_{3}}+w^{\lambda_{3}} w^{\lambda_{1}} w^{\lambda_{2}}+w^{\lambda_{3}} w^{\lambda_{2}} w^{\lambda_{1}}\right) \otimes L_{a},
\end{aligned}
$$

or defining the anti-commutator

$$
\{A, B\} \equiv \frac{1}{2}(A B+B A)
$$

we can express the above representation in a compact form and also find the next level generators:

$$
\begin{aligned}
& L_{a}=1 \otimes L_{a}, \\
& L_{a}^{\lambda_{1}}=-w^{\lambda_{1}} \otimes L_{a}, \\
& L_{a}^{\lambda_{1} \lambda_{2}}=\left\{w^{\lambda_{1}} w^{\lambda_{2}}\right\} \otimes L_{a}, \\
& L_{a}^{\left(\lambda_{1} \lambda_{2}\right) \lambda_{3}}=-\left\{\left\{w^{\lambda_{1}} w^{\lambda_{2}}\right\} w^{\lambda_{3}}\right\} \otimes L_{a}, \\
& L_{a}^{\left(\lambda_{1} \lambda_{2}\right)\left(\lambda_{3} \lambda_{4}\right)}=\left\{\left\{w^{\lambda_{1}} w^{\lambda_{2}}\right\}\left\{w^{\lambda_{3}} w^{\lambda_{4}}\right\}\right\} \otimes L_{a}, \\
& L_{a}^{\left(\left(\lambda_{1} \lambda_{2}\right) \lambda_{3}\right) \lambda_{4}}=\left\{\left\{\left\{w^{\lambda_{1}} w^{\lambda_{2}}\right\} w^{\lambda_{3}}\right\} w^{\lambda_{4}}\right\} \otimes L_{a},
\end{aligned}
$$

This actually means that one should associate the vector variable $e^{\mu}$ with the PauliLubanski vector $e^{\mu} \sim w^{\mu}$ and the generators will take the following general form:

$$
L_{a}^{\lambda_{1} \ldots \lambda_{s}}=\left\{\ldots\left\{w^{\lambda_{1}} \ldots w^{\lambda_{s}}\right\} \otimes L_{a}\right.
$$

where one should understand an appropriate ordering of the non-commuting Pauli-Lubanski vectors, as it is in (45). Using explicit matrix form of the representation (45) we can calculate the commutators of the current subalgebra (11), so that it will take the following form (see Appendix B for the algebraic rules which allow to calculate commutators of the 
matrices given in the form of the Kronecker product) ${ }^{5}$ :

$$
\begin{aligned}
& {\left[L_{a}^{\lambda_{1}}, L_{b}^{\lambda_{2}}\right]=i f_{a b c} L_{c}^{\lambda_{1} \lambda_{2}}-\frac{i}{4} \delta_{a b} \epsilon^{\lambda_{1} \lambda_{2} \rho \sigma} w_{\rho} P_{\sigma},} \\
& \begin{aligned}
& {\left[L_{a}^{\lambda_{1} \lambda_{2}}, L_{b}^{\lambda_{3}}\right]=i f_{a b c} L_{c}^{\left(\lambda_{1} \lambda_{2}\right) \lambda_{3}}+\frac{i}{4} \delta_{a b} }\left(\epsilon^{\lambda_{1} \lambda_{3} \rho \sigma}\left\{w^{\lambda_{2}} w_{\rho}\right\}+\epsilon^{\lambda_{2} \lambda_{3} \rho \sigma}\left\{w^{\lambda_{1}} w_{\rho}\right\}\right) P_{\sigma} \\
& {\left[L_{a}^{\lambda_{1} \lambda_{2}}, L_{b}^{\lambda_{3} \lambda_{4}}\right]=i f_{a b c} L_{c}^{\left(\lambda_{1} \lambda_{2}\right)\left(\lambda_{3} \lambda_{4}\right)}-\frac{i}{4} \delta_{a b}\left(\epsilon^{\lambda_{1} \lambda_{3} \rho \sigma}\left\{w^{\lambda_{2}}\left\{w_{\rho} w^{\lambda_{4}}\right\}\right\}+\epsilon^{\lambda_{2} \lambda_{3} \rho \sigma}\left\{w^{\lambda_{1}}\left\{w_{\rho} w^{\lambda_{4}}\right\}\right\}\right.} \\
&\left.\quad+\epsilon^{\lambda_{1} \lambda_{4} \rho \sigma}\left\{w^{\lambda_{2}}\left\{w_{\rho} w^{\lambda_{3}}\right\}\right\}+\epsilon^{\lambda_{2} \lambda_{4} \rho \sigma}\left\{w^{\lambda_{1}}\left\{w_{\rho} w^{\lambda_{3}}\right\}\right\}\right) P_{\sigma}, \\
& {\left[L_{a}^{\left(\lambda_{1} \lambda_{2}\right) \lambda_{3}}, L_{b}^{\lambda_{4}}\right]=i f_{a b c} L_{c}^{\left(\left(\lambda_{1} \lambda_{2}\right) \lambda_{3}\right) \lambda_{4}}-\frac{i}{4} \delta_{a b}\left(\epsilon^{\lambda_{1} \lambda_{4} \rho \sigma}\left\{\left\{w^{\lambda_{2}} w_{\rho}\right\} w^{\lambda_{3}}\right\}+\epsilon^{\lambda_{2} \lambda_{4} \rho \sigma}\left\{\left\{w^{\lambda_{1}} w_{\rho}\right\} w^{\lambda_{3}}\right\}\right.} \\
&\left.+\epsilon^{\lambda_{3} \lambda_{4} \rho \sigma}\left\{\left\{w^{\lambda_{1}} w^{\lambda_{2}}\right\} w_{\rho}\right\}\right) P_{\sigma},
\end{aligned}
\end{aligned}
$$

where, as we already explained above, the source of this deformation of the current subalgebra (11) is a non-commutativity of the matrices $m_{12}$ and $\pi$. In covariant formulation we can clearly see that the deformation of the current subalgebra appears because the Pauli-Lubanski vector $w^{\mu}$ is a noncommutative vector - its components (43) are not commuting (42). The appearance of the momentum operator in the commutators between tensor generators $L_{a}^{\lambda_{1} \ldots \lambda_{s}}$ in the current subalgebra is reminiscent of the super-Poincaré algebra. The "central"-like terms ${ }^{6}$ can be evaluated further and, in particular,

$$
\delta_{a b} C^{\lambda_{1} \lambda_{2}}=-\frac{i}{4} \delta_{a b} \epsilon^{\lambda_{1} \lambda_{2} \rho \sigma} w_{\rho} P_{\sigma}=-\frac{i}{2} \delta_{a b}\left(P^{2} M^{\lambda_{1} \lambda_{2}}-M^{\lambda_{1} \rho} P_{\rho} P^{\lambda_{2}}+M^{\lambda_{2} \rho} P_{\rho} P^{\lambda_{1}}\right) .
$$

It is interesting to see how the Jacoby identities are satisfied in this case. One can see that

$$
\begin{gathered}
0=\left[\left[L_{a}^{\lambda_{1} \ldots \lambda_{i}}, L_{b}^{\lambda_{i+1} \ldots \lambda_{s}}\right], P^{\mu}\right]+\left[\left[L_{b}^{\lambda_{i+1} \ldots \lambda_{s}}, P^{\mu}\right], L_{a}^{\lambda_{1} \ldots \lambda_{i}}\right]+\left[\left[P^{\mu}, L_{a}^{\lambda_{1} \ldots \lambda_{i}}\right], L_{b}^{\lambda_{i+1} \ldots \lambda_{s}}\right]= \\
=\left[\left[L_{a}^{\lambda_{1} \ldots \lambda_{i}}, L_{b}^{\lambda_{i+1} \ldots \lambda_{s}}\right], P^{\mu}\right]
\end{gathered}
$$

therefore the central term $C^{\lambda_{1} \lambda_{2}}$ should commute with the momentum operator, which indeed takes place.

The main question which remains to be answered is if we can retain the transversal character of the above representation and at the same time avoid a non-commutativity of the Pauli-Lubanski vector and as consequence the corresponding central-like terms. This can be achieved if one considers the derivative of the Pauli-Lubanski vector $w^{\mu}$ over its length - the Casimir invariant $\varrho(63),(64)$. Both operators $\pi^{\prime}$ and $\pi^{\prime \prime}$ are linear functions in $\varrho$ and the operator $m_{12}$ is $\varrho$ - independent (see Appendix A), therefore the derivative will have only transversal components. Thus we shall take the vector variable as follows:

$$
e^{\mu}=\partial_{\varrho} w^{\mu}=\left(0,-\partial_{\varrho} \pi^{\prime \prime}, \partial_{\varrho} \pi^{\prime}, 0\right)=\left(0,-\hat{\pi}^{\prime \prime}, \hat{\pi}^{\prime}, 0\right),
$$

we shall denote it as $\hat{w}^{\mu}$ (see Appendix A):

$$
e^{\mu}=\hat{w}^{\mu}=\left(0,-\hat{\pi}^{\prime \prime}, \hat{\pi}^{\prime}, 0\right)
$$

\footnotetext{
${ }^{5}$ For simplicity we take the generators of the Lie algebra $L(G)$ in the fundamental representation of $\mathrm{SU}(2),\left\{L_{a}, L_{b}\right\}=\frac{1}{4} \delta_{a b}$.

${ }^{6}$ These general formulas for the "central" terms identically coincide with (37) and (41).
} 
This vector has all required properties:

$\alpha)$ it is a commuting vector: $\left[\hat{w}^{\mu}, \hat{w}^{\nu}\right]=0$,

$\beta)$ it is translationally invariant: $\left[P^{\mu}, \hat{w}^{\nu}\right]=0$,

$\gamma$ ) it is a transversal vector: $P^{\mu} \hat{w}_{\mu}=0$,

$\delta$ ) it is a unit space-like vector: $\hat{w}^{2}=-1$.

Thus we shall define the transversal representation as

$$
L_{a}^{\perp \lambda_{1} \ldots \lambda_{s}}=\hat{w}^{\lambda_{1}} \ldots \hat{w}^{\lambda_{s}} \otimes L_{a} .
$$

These generators have components only in the plane transversal to the momentum, the property of the main importance. The generators fulfill the current subalgebra (11) without deformation, because the vector variable $\hat{w}_{\mu}$ is a commuting operator. Having in hand the explicit matrix representations of the extended Poincaré algebra $\mathcal{P}(44),(45),(46)$ and (50) we can calculate different traces which are essential components of the Lagrangian in the extended Yang-Mills theory $[4,5,6]$.

\section{$5 \quad$ Killing Form}

Using the explicit matrix representation of the generators (31) we can compute now the traces (see Appendix C). The matrix of products has the form

$$
<s\left|\operatorname{tr}\left(L_{a}^{\mu} L_{b}^{\nu}\right)\right| s>=\delta_{a b}\left(s^{2} \frac{k^{\mu} k^{\nu}}{\omega^{2}}-\frac{\varrho^{2}}{2}\left(\eta^{\mu \nu}-\frac{\left(k^{\mu} \bar{k}^{\nu}+\bar{k}^{\mu} k^{\nu}\right.}{k \bar{k}}\right)\right)
$$

where $k^{\mu}=(\omega, 0,0, \omega), \bar{k}^{\mu}=(\omega, 0,0,-\omega)$. Having the explicit form for the second level generators (36) we can calculate also the traces:

$$
<s\left|\operatorname{tr}\left(L_{a} L_{b}^{\mu \nu}\right)\right| s>=\delta_{a b}\left(s^{2} \frac{k^{\mu} k^{\nu}}{\omega^{2}}-\frac{\varrho^{2}}{2}\left(\eta^{\mu \nu}-\frac{\left(k^{\mu} \bar{k}^{\nu}+\bar{k}^{\mu} k^{\nu}\right.}{k \bar{k}}\right)\right) .
$$

One can observe that in this formulas the term proportional to $\varrho^{2}$ is multiplied by the projector $\bar{\eta}^{\lambda_{1} \lambda_{2}}$ into the two-dimensional plane transversal to the momentum $k^{\mu}[1]$ :

$$
\bar{\eta}^{\lambda_{1} \lambda_{2}}=-\eta^{\lambda_{1} \lambda_{2}}+\frac{k^{\lambda_{1}} \bar{k}^{\lambda_{2}}+\bar{k}^{\lambda_{1}} k^{\lambda_{2}}}{k \bar{k}}, \quad k_{\lambda_{1}} \bar{\eta}^{\lambda_{1} \lambda_{2}}=k_{\lambda_{2}} \bar{\eta}^{\lambda_{1} \lambda_{2}}=0 .
$$

Because the parameter $\varrho$ is an invariant quantity we can differentiate the trace formula over $\varrho$ in order to get invariant products:

$$
\partial_{\varrho}^{2}<s\left|\operatorname{tr}\left(L_{a}^{\lambda_{1}} L_{b}^{\lambda_{2}}\right)\right| s>=\partial_{\varrho}^{2}<s\left|\operatorname{tr}\left(L_{a} L_{b}^{\lambda_{1} \lambda_{2}}\right)\right| s>=\delta_{a b} \bar{\eta}^{\lambda_{1} \lambda_{2}} .
$$

Having in hand the explicit expressions for the generators (44) and (45) we can also calculate the traces for high order generators:

$$
\begin{gathered}
\partial_{\varrho}^{4}<s\left|\operatorname{tr}\left(L_{a}^{\lambda_{1} \lambda_{2}} L_{b}^{\lambda_{3} \lambda_{4}}\right)\right| s>=\partial_{\varrho}^{4}<s\left|\operatorname{tr}\left(L_{a}^{\left(\lambda_{1} \lambda_{2}\right) \lambda_{3}} L_{b}^{\lambda_{4}}\right)\right| s>= \\
=\partial_{\varrho}^{4}<s\left|\operatorname{tr}\left(L_{a}^{\left(\lambda_{1} \lambda_{2}\right)\left(\lambda_{3} \lambda_{4}\right)} L_{b}\right)\right| s>=\partial_{\varrho}^{4}<s\left|\operatorname{tr}\left(L_{a}^{\left(\left(\lambda_{1} \lambda_{2}\right) \lambda_{3}\right) \lambda_{4}} L_{b}\right)\right| s>= \\
=3 \delta_{a b}\left\{\bar{\eta}^{\lambda_{1} \lambda_{2}} \bar{\eta}^{\lambda_{3} \lambda_{4}}+\bar{\eta}^{\lambda_{1} \lambda_{3}} \bar{\eta}^{\lambda_{2} \lambda_{4}}+\bar{\eta}^{\lambda_{1} \lambda_{4}} \bar{\eta}^{\lambda_{2} \lambda_{3}}\right\} .
\end{gathered}
$$


All generators $L_{a}^{\lambda_{1} \ldots . \lambda_{s}}(44),(45)$ and (46) on a given level $s$ are ordered polynomials of the non-commutative (42) Pauli-Lubanski vectors (45). But when the indices $\lambda_{1}, \ldots ., \lambda_{s}$ run in the transverse plane $\lambda_{1}, \ldots, \lambda_{s}=1,2$ then the components of this vector commute with each other ${ }^{7}\left[w^{1}, w^{2}\right]=\left[-\pi^{\prime \prime}, \pi^{\prime}\right]=0$. Thus the transversal components of the generator are polynomials in the commuting matrices $\pi^{\prime}$ and $\pi^{\prime \prime}$ and therefore are insensitive to the ordering. As a result all generators on a given level $s$ have equal transversal components and therefore all traces in the last formula are equal to each other. In order to illustrate this general structure of the generators let us present their explicit form for the lower rank generators constructed above. They are

$$
\begin{array}{llll} 
& L_{a}^{11}=+\pi^{\prime \prime} \pi^{\prime \prime} \otimes L_{a} & L_{a}^{(11) 1}=+\pi^{\prime \prime} \pi^{\prime \prime} \pi^{\prime \prime} \otimes L_{a} \\
L_{a}^{1}=+\pi^{\prime \prime} \otimes L_{a} & L_{a}^{12}=-\pi^{\prime} \pi^{\prime \prime} \otimes L_{a} & L_{a}^{(12) 1}=-\pi^{\prime} \pi^{\prime \prime} \pi^{\prime \prime} \otimes L_{a} \\
L_{a}^{2}=-\pi^{\prime} \otimes L_{a} & L_{a}^{(12) 2}=L_{a}^{(22) 1}=+\pi^{\prime} \pi^{\prime} \pi^{\prime \prime} \otimes L_{a} \\
& L_{a}^{22}=+\pi^{\prime} \pi^{\prime} \otimes L_{a} & L_{a}^{(22) 2}=-\pi^{\prime} \pi^{\prime} \pi^{\prime} \otimes L_{a}
\end{array} .
$$

In general, the transversal components of the generator $L_{a}^{\lambda_{1} \ldots . \lambda_{s}}$ have the following structure:

$$
L_{a}^{1 \ldots 1} \overbrace{2 \ldots 2}^{k}=\left(\pi^{\prime \prime}\right)^{k}\left(-\pi^{\prime}\right)^{m} \otimes L_{a},
$$

where $k+m=s$ and is therefore proportional to $\varrho^{s}(68)$. The components of the generator $L_{a}^{\lambda_{1} \ldots \lambda_{s}}$ (46) with indices $\lambda_{1} \ldots \lambda_{s}$ within which there are one or more longitudinal coordinates $\lambda=0,3$ will have instead of $\pi$ generators the $m_{12}$ generator, which does not carry the parameter $\varrho$. These longitudinal components carry less power of the parameter $\varrho^{h}$, where $h<s$. This observation has an important consequence, because it follows that the s-order derivative of the generator $L_{a}^{\lambda_{1} \ldots \lambda_{s}}$ over $\varrho$ will "filter out" only transversal components. We shall define transversal generators $L_{a}^{\perp \lambda_{1} \ldots . \lambda_{s}}$ as s-order derivative of the generator $L_{a}^{\lambda_{1} \ldots \lambda_{s}}$ over $\varrho$ :

$$
L_{a}^{\perp \lambda_{1} \ldots \lambda_{s}}=\partial_{\varrho}^{s} L_{a}^{\lambda_{1} \ldots \lambda_{s}}=\partial_{\varrho} w^{\lambda_{1}} \ldots \partial_{\varrho} w^{\lambda_{s}} \otimes L_{a}=\hat{w}^{\lambda_{1}} \ldots \hat{w}^{\lambda_{s}} \otimes L_{a} .
$$

Thus we have seen that analysis of the traces leads us to the same expression for purely transversal representation $(30),(50)$ and $(56)$ of the algebra $L(\mathcal{P})$.

We can now calculate the product of two transversal generators of the total rank $2 n$ :

$$
L_{a}^{\perp \lambda_{1} \ldots \lambda_{i}} L_{b}^{\perp \lambda_{i+1} \ldots \lambda_{2 n}}
$$

Its components are the aggregates of $\hat{\pi}$ matrices, so that calculating the corresponding traces we come up with the general formula for the Killing form:

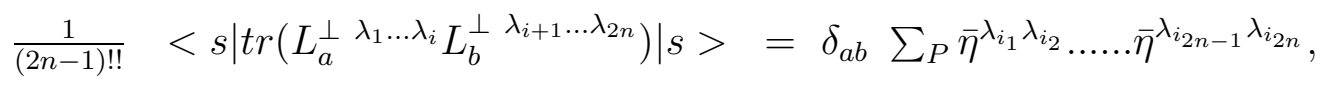

which contains a symmetric product of projector (52) [1]. This traces are an essential ingredients of the Lagrangian, which is a quadratic form in field strength tensor $[4,5,6]$.

\footnotetext{
${ }^{7}$ These are transversal indices, that is, the components of the generator which are transversal to the vector $k^{\mu}=(\omega, 0,0, \omega)$.
} 


\section{Conclusion}

In our studies of a generalization of the Yang-Mills theory it has been found that the group of gauge transformations gets essentially enlarged $[4,5,6]$. This enlargement involves an elegant mixture of the internal and space-time symmetries. The resulting group is an extension of the Poincaré group with an infinitely many generators which carry internal and space-time indices $[7,8]$. Our main concern in this article was to study the irreducible representation of this group and its relation with the generalization of the Yang-Mills theory.

We have found two essentially different representations of the algebra $L(\mathcal{P})$. The first one can be characterized as longitudinal representation, because its tensor generators $L_{a}^{\| \lambda_{1} \ldots \lambda_{s}}$ are built up as symmetric polynomials of momentum (29)

$$
L_{a}^{\| \lambda_{1} \ldots \lambda_{s}}=k^{\lambda_{1}} \ldots k^{\lambda_{s}} \otimes L_{a}
$$

and are obviously divergentless: $k_{\lambda_{1}} L_{a}^{\| \lambda_{1} \ldots \lambda_{s}}=0$. If the gauge fields take value in this representation of the algebra $\mathcal{P}$ then they reduce to a collection of the Yang-Mills fields $B_{\mu}^{a}$ of the form

$$
A_{\mu \lambda_{1} \ldots \lambda_{s}}^{a} L_{a}^{\| \lambda_{1} \ldots \lambda_{s}}
$$

where each of these tensor fields carries the highest helicity $h= \pm 1$. There are no high spin states in this representation of $\mathcal{P}$ and, as a consequence, in the corresponding gauge theory.

The second, the transversal representation of the generators $L_{a}^{\perp \lambda_{1} \ldots \lambda_{s}}$ is build up as symmetric polynomials of the vector variable $\hat{w}^{\mu}$ which is a derivative of the Pauli-Lubanski vector over its length (49), (50):

$$
L_{a}^{\perp \lambda_{1} \ldots \lambda_{s}}=\hat{w}^{\lambda_{1}} \ldots \hat{w}^{\lambda_{s}} \otimes L_{a} .
$$

These symmetric generators are transversal $k_{\lambda_{1}} L_{a}^{\perp \lambda_{1} \ldots \lambda_{s}}=0$, space-like tensors carrying the helicities:

$$
\pm s, \quad \pm(s-2), \quad \pm(s-4), \ldots
$$

because each vector $\hat{w}^{\lambda_{i}}$ is a transversal $k_{\mu} \hat{w}^{\mu}=0$ and purely spatial unit vector $\hat{w}^{2}=-1$, which carries a non-zero helicities $h= \pm 1$. Therefore the generators $L_{a}^{\perp \lambda_{1} \ldots \lambda_{s}}$ are projecting out the components of the non-Abelian tensor gauge field $A_{\mu \lambda_{1} \ldots \lambda_{s}}^{a}$ into the plane transversal to the momentum:

$$
A_{\mu \lambda_{1} \ldots \lambda_{s}}^{a} L_{a}^{\perp \lambda_{1} \ldots \lambda_{s}}
$$

keeping only its positively definite space-like components of helicities:

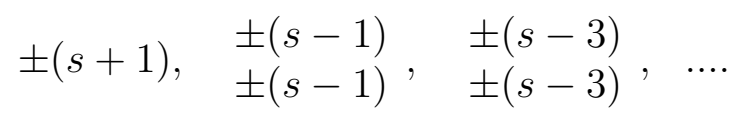

where the lower helicity states have double degeneracy. This can be seen in the frame which is associated with the fixed momentum $k^{\mu}=\omega(1,0,0,1)$, so that the tensor gauge fields describe transversal, positive norm states of the highest helicity $s+1$. Because the whole construction is based on the representation of the space-time group $\mathcal{P}$ the above statement is relativistically invariant (see also the Appendix A). 
In conclusion one can suggest the following geometrical interpretation of the group $\mathcal{P}$ and of the corresponding gauge field theory $[4,5,6]$. In the Yang-Mills theory [14, 15] with each point of the space-time is associated a vector space $V_{x}$ of charged spinor fields $\psi$, on which the action of the Lie group $\mathrm{G}$ is defined as $\psi^{\prime}=U_{\xi} \psi$. The gauge field $A_{\mu}$ is a connection which defines a parallel transport of $\psi: \delta \psi=i g A_{\mu} \psi d x^{\mu}$ and is a Lie algebra valued 1 -form.

In the extended Yang-Mills theory, with each space-time point $\mathrm{x}$ one should associate a vector space $\mathcal{V}_{x}$ of charged Rarita-Schwinger tensor-spinors fields $\psi_{\lambda_{1} \ldots \lambda_{s}} \hat{w}^{\lambda_{1}} \ldots \hat{w}^{\lambda_{s}}[5$, $27,28,29]$, on which the action of the group $\mathcal{P}$ is given by the group element $U_{\xi}=$ $\exp \left(\sum \frac{1}{s !} \xi_{\lambda_{1} \ldots \lambda_{s}}^{a} L_{a}^{\perp \lambda_{1} \ldots \lambda_{s}}\right)$. The extended gauge field $\mathcal{A}(x, L)$ is a connection which defines a parallel transport of spinor-tensors and is a $L(\mathcal{P})$ algebra valued 1 -form. The algebra $L(\mathcal{P})$ is not a purely internal algebra - it is a mixture of internal and space-time algebras, which carries not only internal charges, but also a nonzero helicities. The group $\mathcal{P}$ acts simultaneously as a structure group on the fibers and as an isometry group of the base manifold.

I would like to thank Prof.Ludwig Faddeev for stimulating discussions and his interpretation of the algebra (1) as an example of a current algebra.

\section{Appendix A. Representations of the Poinceré Group}

The Poincaré group has an invariant subgroup, consisting of all displacements $U_{a}=$ $\exp \left(i a_{\mu} P^{\mu}\right)$, and it is Abelian group. Since the group of displacements is Abelian, the unitary irreducible representations are one-dimensional, $U_{a}|k>=\exp (-i k \cdot a)| k>$, where $a$ is the displacement vector and the vector $\mid k>$ describes a state of the Hilbert space with momentum $k^{\mu}$. If the representation of the Poincaré group contains the representation $\exp (-i k \cdot a)$ of the displacement group, it also contains all representations $\exp \left(-i k^{\prime} \cdot a\right)$ of this subgroup with $k^{\prime}=\theta k$ obtainable from $k$ by a proper Lorentz transformation $\theta_{\mu \nu}$. Indeed, since $\mid k>$ describes a state of the Hilbert space with momentum $k^{\mu}$ it follows that the operation $U_{\theta}=\exp \left(\frac{i}{2} \theta_{\mu \nu} M^{\mu \nu}\right)$, which corresponds to the Lorentz transformation $\theta_{\mu \nu}$, will transform the state $\mid k>$ into the state with momentum $k^{\prime}=\theta k$

$$
U_{a}\left(U_{\theta} \mid k>\right)=\exp (-i \theta k \cdot a) U_{\theta} \mid k>
$$

It is a consequence of the equations $U_{a} U_{\theta}=U_{\theta} U_{\theta^{-1} a}$ and $\quad k \cdot \theta^{-1} a=\theta k \cdot a$. Thus if the representation of the Poincaré group contains the state $\mid k>$ with momentum $k$, it also contains states with all the momentum $k^{\prime}=\theta k$, where $\theta_{\mu \nu}$ is any Lorentz transformation. Thus, in an irreducible representation, all such states can be obtained from a single one $\mid k>$ by applying to it all possible Lorentz transformations $U_{\theta} \mid k>$. The length $k^{2}$ of the momenta is the same for all state vectors which are present in the representation space of an irreducible representation.

All Lorentz transformations $\theta_{\mu \nu}$ which leave a fixed null vector $k^{\mu}=\omega(1,0,0,1)$ invariant $\theta k=k$ form a subgroup called "little group" [23]. The group of Lorentz transformations which leave a null vector $k^{\mu}$ invariant is a two-dimensional Euclidean group of rotations $U(\theta)=\exp \left(-i m_{12} \theta\right)$ in the plane transverse to the vector $\vec{k}=(0,0,1)$ and displacements $U^{\prime}(\alpha)=\exp \left(-i \alpha \pi^{\prime}\right), U^{\prime \prime}(\beta)=\exp \left(-i \beta \pi^{\prime \prime}\right)$, which are induced by Lorentz generators

$$
m_{12}=M_{12}, \quad \pi^{\prime}=M_{10}+M_{13}, \quad \pi^{\prime \prime}=M_{20}+M_{23}
$$


They form the Euclidean algebra $E(2)$

$$
\left[\pi^{\prime}, \pi^{\prime \prime}\right]=0, \quad\left[m_{12}, \pi^{\prime}\right]=i \pi^{\prime \prime}, \quad\left[m_{12}, \pi^{\prime \prime}\right]=-i \pi^{\prime} .
$$

The irreducible unitary representation of the little group uniquely defines the representation of the Poinceré group and it does not depend on the arbitrary choice of the fixed null vector $k^{\mu}=\omega(1,0,0,1)[23]$.

Two Casimir operators of the Poincaré group are given by the operators $P^{2}$ and $w^{2}-$ square of the Pauli-Lubanski vector $2 w^{\mu}=\epsilon^{\mu \nu \lambda \rho} P_{\nu} M_{\lambda \rho}=\epsilon^{\mu \nu \lambda \rho} M_{\nu \lambda} P_{\rho}$

$$
-w^{2}=\pi^{\prime 2}+\pi^{\prime \prime 2}
$$

where $w^{\mu}$ in components is

$$
w^{\mu}=\left(-m_{12},-\pi^{\prime \prime}, \pi^{\prime},-m_{12}\right) .
$$

To describe representations of the little group one can take $m_{12}$ in a diagonal form with integer eigenvalues $s=0, \pm 1, \pm 2, \ldots$ and then from Lorentz subalgebra (61) it follows that both of the $\Pi$ generators have Jacoby form [23]:

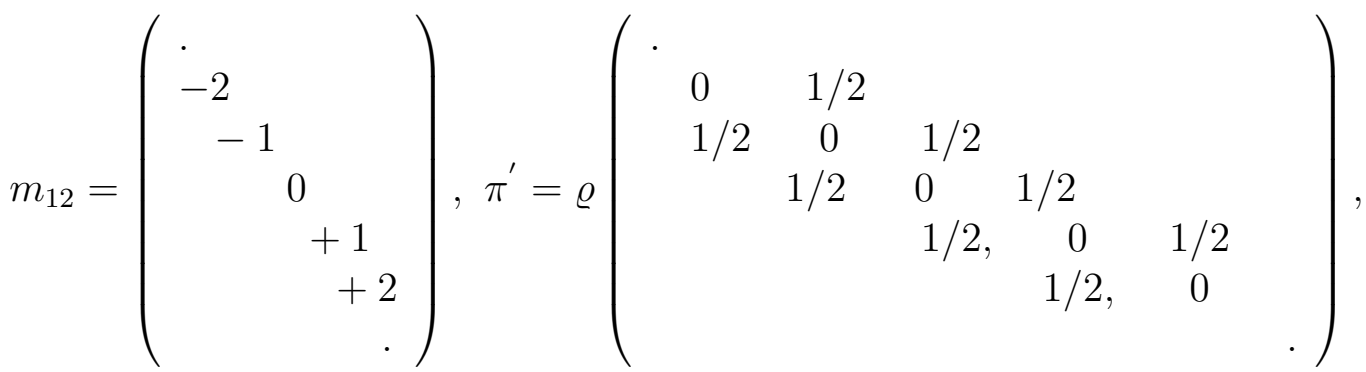

$$
\begin{aligned}
& \pi^{\prime \prime}=\varrho\left(\begin{array}{crrrr}
0 & i / 2 & & & \\
-i / 2 & 0 & i / 2 & & \\
& -i / 2 & 0 & i / 2 & \\
& & -i / 2, & 0 & i / 2 \\
& & & -i / 2, & 0
\end{array}\right)
\end{aligned}
$$

These infinitely dimensional representations can be characterized by the parameter $\varrho$ which can be assumed to be any positive real number. One can now compute the Casimir operator for these representations:

$$
-w^{2}=\pi^{\prime 2}+\pi^{\prime \prime 2}=\varrho^{2} .
$$

For our purposes it is convenient to define operators $\hat{\pi}^{\prime}=\pi^{\prime} / \varrho$ and $\hat{\pi}^{\prime \prime}=\pi^{\prime \prime} / \varrho$, so that

$$
\hat{\pi}^{2}+\hat{\pi}^{\prime \prime 2}=1
$$

and we shall have

$$
\hat{w}^{\mu}=\partial_{\varrho} w^{\mu}=\left(0,-\partial_{\varrho} \pi^{\prime \prime}, \partial_{\varrho} \pi^{\prime}, 0\right)=\left(0,-\hat{\pi}^{\prime \prime}, \hat{\pi}^{\prime}, 0\right) .
$$


Let us define the state with fixed helicity $s(0, \pm 1, \pm 2, \ldots)$ as

$$
m_{12}|s>=s| s>\text {. }
$$

It can be represented as an infinite vector with entry one in the row s:

$$
\mid s>=\left(\begin{array}{c}
. \\
0 \\
1 \\
0 \\
0 \\
\cdot
\end{array}\right)
$$

The action of the $\pi^{\prime}$ generators on this vector can be found by using (63):

$$
\pi^{\prime}\left|s>=\frac{\varrho}{2}(|s-1>+| s+1>), \quad \pi^{\prime \prime}\right| s>=\frac{i \varrho}{2}(|s-1>-| s+1>) .
$$

These relations tell us that under the Lorentz boosts the state with helicity $\mid s>$ transforms into the states with helicities $\mid s \pm 1>$ with the amplitude proportional to $\varrho / 2$.

In the case when $\varrho=0$ we shall have invariant pure helicity states $\mid s>$ which decouple from each other. These are massless representations $\mathbf{0}_{\mathbf{s}}$ of the Poincaré algebra. When $\varrho \neq$ 0 we shall have representations, in which helicity takes any integer value $s=0, \pm 1, \pm 2, \ldots$. These are so called massless "continuous spin representations" - $\mathbf{0}_{\varrho}$ of the Poincaré algebra [23]. Let us introduce the coherent state of different helicities $|\varphi\rangle=\sum_{s} e^{i s \varphi}|s\rangle$. Under rotation $U(\theta)$ in xy-plane it will transform as

$$
U(\theta)\left|\varphi>=\sum_{s} e^{i s \varphi} U(\theta)\right| s>=\sum_{s} e^{i s \varphi} e^{i s \theta}|s>=| \varphi+\theta>
$$

and under Lorentz boosts as

$$
\pi^{\prime}\left|\varphi>=\sum_{s} e^{i s \varphi} \Pi^{\prime}\right| s>=\sum_{s} e^{i s \varphi} \frac{\varrho}{2}(|s-1>+| s+1>)=\varrho \cos \varphi \mid \varphi>
$$

and $\pi^{\prime \prime}|\varphi>=\varrho \sin \varphi| \varphi>$. Thus we have the following representation of the little algebra (61):

$$
m_{12}=-i \frac{\partial}{\partial \varphi}, \quad \pi^{\prime}=\varrho \cos \varphi, \quad \pi^{\prime \prime}=\varrho \sin \varphi
$$

\section{Appendix B. The Kronecker product}

The Kronecker product, defined by $\otimes$, is an operation on two matrices of arbitrary size resulting in a block matrix

$$
A \otimes B=\left(\begin{array}{lll}
a_{11} B & \ldots & a_{1 n} B \\
\ldots & \ldots & \ldots \\
a_{n 1} B & \ldots & a_{n n} B
\end{array}\right)
$$

It has mixed-product property. For matrices $\mathrm{A}, \mathrm{B}, \mathrm{C}$ and $\mathrm{D}$, for which the matrix product $A C$ and $B D$ is defined, one has

$$
(A \otimes B)(C \otimes D)=A C \otimes B D .
$$


This property allows simple calculation of the commutator:

$$
\begin{aligned}
{[A \otimes B, C \otimes D] } & =[A, C] \otimes B D+C A \otimes[B, D]= \\
& =A C \otimes[B, D]+[A, C] \otimes D B= \\
& =[A, C] \otimes \frac{1}{2}(B D+D B)+\frac{1}{2}(A C+C A) \otimes[B, D] \\
& =[A, C] \otimes\{B D\}+\{A C\} \otimes[B, D],
\end{aligned}
$$

where

$$
\{A, C\} \equiv \frac{1}{2}(A C+C A) .
$$

\section{Appendix C. Calculation of Killing Form}

Using the explicit matrix representation of the generators (31) and representations (63) or (70) we can compute the traces

$$
\begin{gathered}
<s\left|\operatorname{tr}\left(L_{a}^{0} L_{b}^{0}\right)\right| s>=\delta_{a b} s^{2}, \quad<s\left|\operatorname{tr}\left(L_{a}^{0} L_{b}^{3}\right)\right| s>=\delta_{a b} s^{2}, \quad<s\left|\operatorname{tr}\left(L_{a}^{3} L_{b}^{3}\right)\right| s>=\delta_{a b} s^{2}, \\
<s\left|\operatorname{tr}\left(L_{a}^{1} L_{b}^{1}\right)\right| s>=\delta_{a b} \frac{o^{2}}{2}, \quad<s\left|\operatorname{tr}\left(L_{a}^{2} L_{b}^{2}\right)\right| s>=\delta_{a b} \frac{a^{2}}{2} .
\end{gathered}
$$

All other traces are equal to zero. Thus the matrix of products has the following form:

$$
\begin{aligned}
<s\left|\operatorname{tr}\left(L_{a}^{\mu} L_{b}^{\nu}\right)\right| s> & =\delta_{a b}\left(\begin{array}{cccc}
s^{2} & 0 & 0 & s^{2} \\
0 & \frac{\varrho^{2}}{2} & 0 & 0 \\
0 & 0 & \frac{\varrho^{2}}{2} & 0 \\
s^{2} & 0 & 0 & s^{2}
\end{array}\right) \\
& =\delta_{a b}\left(s^{2} \frac{k^{\mu} k^{\nu}}{\omega^{2}}-\frac{\varrho^{2}}{2}\left(\eta^{\mu \nu}-\frac{\left(k^{\mu} \bar{k}^{\nu}+\bar{k}^{\mu} k^{\nu}\right.}{2 \omega^{2}}\right)\right) \\
& =\delta_{a b}\left(s^{2} \frac{k^{\mu} k^{\nu}}{\omega^{2}}+\frac{\varrho^{2}}{2} \sum_{\lambda=1,2} e_{\lambda}^{\mu} e_{\lambda}^{\nu}\right),
\end{aligned}
$$

where $k^{\mu}=(\omega, 0,0, \omega), \bar{k}^{\mu}=(\omega, 0,0,-\omega)$ and $e_{1}^{\mu}=(0,1,0,0), e_{2}^{\mu}=(0,0,1,0)$. Having the explicit form of the second level generators (36) we can calculate the other traces as well:

$$
<s\left|\operatorname{tr}\left(L_{a} L_{b}^{\mu \nu}\right)\right| s>=\delta_{a b}\left(\begin{array}{cccc}
s^{2} & 0 & 0 & s^{2} \\
0 & \frac{\varrho^{2}}{2} & 0 & 0 \\
0 & 0 & \frac{\underline{\rho}^{2}}{2} & 0 \\
s^{2} & 0 & 0 & s^{2}
\end{array}\right) .
$$

One can observe that in this formulas the term proportional to $\varrho^{2}$ is multiplied by the projector $\bar{\eta}^{\lambda_{1} \lambda_{2}}$ into the two-dimensional plane transversal to the momentum $k^{\mu}$ [1]:

$$
\bar{\eta}^{\lambda_{1} \lambda_{2}}=-\eta^{\lambda_{1} \lambda_{2}}+\frac{k^{\lambda_{1}} \bar{k}^{\lambda_{2}}+\bar{k}^{\lambda_{1}} k^{\lambda_{2}}}{k \bar{k}}, \quad k_{\lambda_{1}} \bar{\eta}^{\lambda_{1} \lambda_{2}}=k_{\lambda_{2}} \bar{\eta}^{\lambda_{1} \lambda_{2}}=0 .
$$

Because the parameter $\varrho$ is an invariant quantity we can differentiate the traces over the parameter $\varrho$ to get the following important formulas:

$$
\partial_{\varrho}^{2}<s\left|\operatorname{tr}\left(L_{a}^{\lambda_{1}} L_{b}^{\lambda_{2}}\right)\right| s>=\partial_{\varrho}^{2}<s\left|\operatorname{tr}\left(L_{a} L_{b}^{\lambda_{1} \lambda_{2}}\right)\right| s>=\delta_{a b} \bar{\eta}^{\lambda_{1} \lambda_{2}} .
$$




\section{Appendix D. Virasoro-Kac-Moody Algebra}

This algebra is defined by the commutators

$$
\begin{aligned}
& {\left[T^{n}, T^{m}\right]=(n-m) T^{n+m},} \\
& {\left[T^{n}, L_{a}^{m}\right]=-m L_{a}^{n+m},} \\
& {\left[L_{a}^{n}, L_{b}^{m}\right]=i f_{a b c} L_{c}^{n+m}}
\end{aligned}
$$

and has a simple representation: $T^{n}=-z^{n} \frac{d}{d z}, \quad L_{a}^{n}=L_{a} z^{n}$, where $z$ is a complex variable on the two-dimensional "space-time" plane. It can be considered as an analogue of the proposed extension of the Poincaré algebra (12) in a sense that the new vector variable $e_{\lambda}$ and the complex variable $z$ play a similar role, so that the Virasoro subalgebra can be associated with the Poincaré subalgebra and Kac-Moody subalgebra with the "current" subalgebra $(11)^{8}$.

\section{References}

[1] J.Schwinger, Particles, Sourses, and Fields, Vol.1 (Addison-Wesley, Reading, MA, 1970)

[2] S. R. Coleman and J. Mandula, All possible symmetries of the $S$ matrix, Phys. Rev. 159 (1967) 1251.

[3] R. Haag, J. T. Lopuszanski and M. Sohnius, All Possible Generators Of Supersymmetries Of The S Matrix, Nucl. Phys. B 88 (1975) 257.

[4] G. Savvidy, Non-Abelian tensor gauge fields: Generalization of Yang-Mills theory, Phys. Lett. B 625 (2005) 341

[5] G. Savvidy, Non-abelian tensor gauge fields. I, Int. J. Mod. Phys. A 21 (2006) 4931;

[6] G. Savvidy, Non-abelian tensor gauge fields. II, Int. J. Mod. Phys. A 21 (2006) 4959;

[7] G. Savvidy, Interaction of non-Abelian tensor gauge fields, Arm. J. Math. 1 (2008) 1 [arXiv:0804.2003 [hep-th]].

[8] G. Savvidy, Non-Abelian Tensor Gauge Fields, arXiv:1004.4456 [hep-th].

[9] Yu. A. Golfand and E. P. Likhtman, Extension of the Algebra of Poincare Group Generators and Violation of p Invariance, JETP Lett. 13 (1971) 323

[10] P. Ramond, Dual Theory for Free Fermions, Phys. Rev. D 3 (1971) 2415.

[11] A. Neveu and J. H. Schwarz, Factorizable dual model of pions, Nucl. Phys. B 31 (1971) 86.

[12] D. V. Volkov and V. P. Akulov, Is the Neutrino a Goldstone Particle?, Phys. Lett. B 46 (1973) 109.

\footnotetext{
${ }^{8}$ Different extensions of the Poincaré algebra recently have been considered in [30].
} 
[13] J. Wess and B. Zumino, Supergauge Transformations in Four-Dimensions, Nucl. Phys. B 70 (1974) 39.

[14] C.N.Yang and R.L.Mills. Conservation of Isotopic Spin and Isotopic Gauge Invariance. Phys. Rev. 96 (1954) 191

[15] S.S.Chern. Topics in Defferential Geometry, Ch. III "Theory of Connections" (The Institute for Advanced Study, Princeton, 1951)

[16] L. D. Faddeev, Operator Anomaly For The Gauss Law, Phys. Lett. B 145 (1984) 81.

[17] J. K. Barrett and G. Savvidy, A dual lagrangian for non-Abelian tensor gauge fields, Phys. Lett. B 652 (2007) 141

[18] M. Fierz. Über die relativistische Theorie kräftefreier Teilchen mit beliebigem Spin, Helv. Phys. Acta. 12 (1939) 3.

[19] M. Fierz and W. Pauli. On Relativistic Wave Equations for Particles of Arbitrary Spin in an Electromagnetic Field, Proc. Roy. Soc. A173 (1939) 211.

[20] H.Yukawa, Quantum Theory of Non-Local Fields. Part I. Free Fields, Phys. Rev. 77 (1950) 219 ; M. Fierz, Non-Local Fields, Phys. Rev. 78 (1950) 184

[21] E. Wigner, Invariant Quantum Mechanical Equations of Motion, in Theoretical Physics ed. A.Salam (International Atomic Energy, Vienna, 1963) p 59

[22] G. K. Savvidy, Tensionless strings: Physical Fock space and higher spin fields, Int. J. Mod. Phys. A 19 (2004) 3171 [arXiv:hep-th/0310085].

[23] E. Wigner. On Unitary Representations of the Inhomogeneous Lorentz Group. Ann. Math. 40 (1939) 149.

[24] S. Weinberg, Feynman Rules For Any Spin, Phys. Rev. 133 (1964) B1318.

[25] L. P. S. Singh and C. R. Hagen, Lagrangian formulation for arbitrary spin. I. The boson case, Phys. Rev. D9 (1974) 898

[26] C.Fronsdal, Massless fields with integer spin, Phys.Rev. D18 (1978) 3624

[27] W. Rarita and J. Schwinger. On a Theory of Particles with Half-Integral Spin. Phys. Rev. 60 (1941) 61

[28] L. P. S. Singh and C. R. Hagen, Lagrangian formulation for arbitrary spin. II. The fermion case, Phys. Rev. D9 (1974) 898, 910

[29] J.Fang and C.Fronsdal, Massless fields with half-integral spin, Phys. Rev. D18 (1978) 3630

[30] S. Bonanos and J. Gomis, Infinite Sequence of Poincare Group Extensions: Structure and Dynamics, J. Phys. A 43 (2010) 015201 [arXiv:0812.4140 [hep-th]]. 